\title{
Outcome of very preterm infants delivered outside tertiary perinatal centers in China: a multi-center cohort study
}

\author{
Shulin Pan ${ }^{1 \#}$, Siyuan Jiang ${ }^{2 \#}$, Su Lin ${ }^{1}$, Shoo K. Lee ${ }^{3,4,5}$, Yun $\mathrm{Cao}^{2}$, Zhenlang Lin $^{1}$; on behalf of the \\ Reduction of Infection in Neonatal Intensive Care Units using the Evidence-based Practice for \\ Improving Quality (REIN-EPIQ) Study Group
}

\begin{abstract}
${ }^{1}$ Department of Neonatology, The Second Affiliated Hospital \& Yuying Children's Hospital, Wenzhou Medical University, Wenzhou, China; ${ }^{2}$ Department of Neonatology, Children's Hospital of Fudan University, Shanghai, China; ${ }^{3}$ Maternal-Infant Care Research Centre and Department of Pediatrics, Mount Sinai Hospital, Toronto, Ontario, Canada; ${ }^{4}$ Department of Pediatrics, University of Toronto, Toronto, Ontario, Canada; ${ }^{5}$ Department of Obstetrics and Gynecology and Dalla Lana School of Public Health, University of Toronto, Toronto, Ontario, Canada

Contributions: (I) Conception and design: S Pan, S Jiang, Y Cao, Z Lin; (II) Administrative support: Z Lin, Y Cao, SK Lee; (III) Provision of study materials or patients: None; (IV) Collection and assembly of data: S Pan, S Jiang, S Lin; (V) Data analysis and interpretation: S Pan, S Jiang; (VI) Manuscript writing: All authors; (VII) Final approval of manuscript: All authors.

\#These authors contributed equally to this work.

Correspondence to: Yun Cao. Department of Neonatology, Children's Hospital of Fudan University, 399 Wanyuan Road, Minhang District, Shanghai 201102, China. Email: yuncao@fudan.edu.cn; Zhenlang Lin. Department of Neonatology, The Second Affiliated Hospital \& Yuying Children's Hospital, Wenzhou Medical University, Wenzhou 325000, China. Email: linzhenlang@hotmail.com.
\end{abstract}

Background: To describe the incidence of outborns among very preterm infants (VPIs, <32 weeks of gestation) in Chinese perinatal centers and to examine the association of outborn status with adverse outcomes.

Methods: A cohort study enrolling all VPIs admitted to 18 perinatal centers in China from May 1st, 2015 to April 30th, 2018. Neonatal outcomes including rates of discharge against medical advice (DAMA), in-hospital mortality, overall mortality, severe intraventricular hemorrhage (IVH) or periventricular leukomalacia (PVL), sepsis, bronchopulmonary dysplasia (BPD), necrotizing enterocolitis (NEC) and severe retinopathy of prematurity (ROP) were compared between outborn and inborn infants. A multivariate logistic regression model was used to estimate the independent association of outborn status with neonatal outcomes.

Results: Among 12,014 VPIs, 1,991 (16.6\%) infants were outborn. Outborn infants had lower Apgar scores and higher illness severity score on admission. Mothers of outborn infants were less likely to receive antenatal steroids, prenatal care and caesarean section. The incidence of DAMA $(18.0 \%$ vs. $12.5 \%, \mathrm{P}<0.001)$, overall mortality $(19.9 \%$ vs. $15.8 \%, \mathrm{P}<0.001)$ and severe brain injury $(10.8 \%$ vs. $9.1 \%, \mathrm{P}=0.024)$ of outborn infants were significantly higher than inborn infants. Outborn status was independently associated with increased risks of DAMA (aOR, 1.6; 95\% CI: 1.4-1.8), overall-hospital mortality (aOR, 1.3; 95\% CI: 1.1-1.5) and severe IVH/PVL (aOR, 1.2; 95\% CI: 1.0-1.5).

Conclusions: The incidence of outborn VPIs was high in China. Outborn infants were more likely to be delivered in an uncontrolled situation and were at significantly higher risk of neonatal mortality and severe brain injury compared with inborn infants. Quality improvement efforts are needed to facilitate in-utero transfer of high-risk pregnancies to tertiary centers.

Keywords: Preterm infants; outborn; outcome; China

Submitted Aug 07, 2020. Accepted for publication Dec 15, 2020.

doi: $10.21037 / \mathrm{tp}-20-232$

View this article at: http://dx.doi.org/10.21037/tp-20-232 


\section{Introduction}

Babies born at less than 32 (very preterm infants) weeks represent about $16 \%$ of all preterm births, but account for the majority of preterm deaths (1). Many studies from various countries reported better outcomes of preterm infants born in tertiary perinatal centers (inborn) compared with those delivered in hospitals without capability to provide comprehensive care for preterm infants and transferred to tertiary centers for further treatment (outborn) (2-10). Outborn infants were found to have higher risks of mortality and severe brain injury compared with inborn infants (2-4,6,8). In 2002, the American Academy of Pediatrics and American College of Obstetricians and Gynecologists recommended that births at $<32$ weeks' gestational age should occur at subspecialty perinatal centers (11).

Although there have been significant improvements of perinatal care in China, problems remain. More and more preterm infants received active care in NICUs in the recent decades, while the mortality and morbidities of very preterm infants remained high requiring quality improvement of perinatal care practices. Also, the regionalization of care for high risk mothers and the transferring systems of high-risk mothers instead of neonates has not well been established (12). Information about outborn status and outcomes of preterm infants in relation to outborn status has not been reported in China. In our study, we use the largest contemporary cohort of preterm infants born less than 32 weeks' gestation from 18 perinatal centers in China, aiming to describe the incidence of outborns in Chinese perinatal centers, and to compare neonatal outcomes of outborn and inborn infants in China. These data will provide insights to modification of current perinatal care system in China. We present the following article in accordance with the STROBE reporting checklist (available at http://dx.doi.org/10.21037/tp-20-232).

\section{Methods}

\section{Study design and settings}

The current study is a cohort study using data from a clinical database initially established for a cluster randomized controlled study entitled "Reduction of Infection in Neonatal Intensive Care Units using the Evidence-based Practice for Improving Quality (EPIQ)" (REIN-EPIQ study, clinicaltrials.gov \#NCT02600195). Twenty-five hospitals prospectively collected clinical data, including maternal and neonatal characteristics, treatment in the NICU and neonatal outcomes, of all admitted preterm infants using this data base from May 1st, 2015 and April 30th, 2018. All data collection followed a standard manual of operations and definitions (13).

Among the original participating 25 hospitals, 18 hospitals were tertiary perinatal centers and were enrolled in this study. The characteristics of all centers are shown in Table S1. The median annual number of births was 17,000/year (IQR 9,330-21,000/year). The median bed number of NICU beds per hospital was 51 (IQR 30 68 beds). All centers admitted outborn infants except one hospital. Thirteen hospitals had dedicated transport teams to transfer outborn infants from referring hospitals. For perinatal centers without dedicated transport teams, local medical emergency transport systems provided the transportation, however, staff of these general transport systems were not trained in neonatal care and the ambulances were not equipped with incubators and other necessary equipment for neonatal transport. At the time of this study, the majority of transports were by land, very few by railway and no by air. All of the perinatal centers provided comprehensive care for infants with gestational age $<28$ weeks or birth weight $<1,000 \mathrm{~g}$. The study was conducted in accordance with the Declaration of Helsinki (as revised in 2013). The study was approved by the Ethics Committee of the Children's Hospital of Fudan University (NO. 201528) and written informed consent from the patients was not required because this study did not directly intervene in the diagnosis and treatment of individual patients.

\section{Study population}

All infants born at $<32$ weeks' gestation between May 1st, 2015 and April 30th, 2018 and admitted to participating NICUs within 7 days of birth were included in the study. Stillborn and delivery room deaths were not included. Infants were followed until death or discharge from the NICU. If infants were transferred from one to another participating NICU, outcomes were assigned to the NICU of first admission. If infants transferred to non-participating NICUs, data were collected until discharge from the participating unit.

\section{Definitions}

Inborn status was defined as infants born in the eighteen 
perinatal centers participating in our study and subsequent admitted to the NICU of the same hospital. Outborn status was defined as infants born in level I or level II NICUs or hospitals without neonatal care, and transferred to one of the eighteen participating perinatal centers within 7 days after birth. The study included all infants born outside participating tertiary hospitals including those who were born at home.

Gestational age was determined using the hierarchy of best obstetric estimate based on prenatal ultrasound, menstrual history, obstetric examination, or all three. If the obstetric estimate was not available or was different from the postnatal pediatric estimate of gestation by more than two weeks, the gestational age was estimated using the Ballard Score $(14,15)$. Small for gestational age (SGA) was defined as birth weight $<10$ th percentile for the gestational age according to the Chinese neonatal birth weight values (16). Prenatal care was defined as at least one pregnancy-related hospital visit during pregnancy. Antenatal corticosteroid treatment was defined as partial or complete course of antenatal corticosteroids prior to birth. Transport Risk Index of Physiologic Stability (TRIPS) score $(17,18)$ was used as an illness severity score on NICU admission. Maternal diabetes included gestational diabetes, Type 1 diabetes, Type 2 diabetes or diabetes with unknown type. Maternal hypertension included hypertension that was preexisting before current pregnancy, gestational hypertension and hypertension with unknown timing.

Discharged against medical advice (DAMA) was defined as parental choice to terminate treatment to infant before discharge was recommended by a physician. Logistical constraints prevented us from identifying whether infants discharged against medical advice survived, so we used predefined criteria to predict the likelihood of death (13). If DAMA infants required invasive or non-invasive mechanical ventilation, inotropes infusion, or total parenteral nutrition (no enteral feeds initiated) on the day of discharge, we predicted that they would die. Two investigators independently adjudicated the predicted mortality and differences were resolved by consensus.

Sepsis included both culture-proven sepsis and clinical sepsis. Culture-proven sepsis was diagnosed according to Stoll et al. (19). Clinical sepsis was diagnosed when all the following criteria were fulfilled: (I) infection-related clinical manifestations; (II) abnormal white blood cell count (white blood cell $<5 \times 10^{9} / \mathrm{L}$ or $\left.>20 \times 10^{9} / \mathrm{L}\right)$, CRP level $(\geq 8 \mathrm{mg} / \mathrm{L})$, or PCT level ( $>0.5 \mathrm{ng} / \mathrm{mL}$ ); (III) antibiotics used or intended for $\geq 5$ days; (IV) negative blood culture with no or negative cerebrospinal fluid culture; (V) no evidence of concurrent focal infection, including pneumonia, urinary tract infection, and necrotizing enterocolitis. NEC was defined as $\geq$ stage 2 according to Bell's criteria (13). IVH was defined as $\geq$ grade 3 according to Papile's criteria (20) or periventricular leukomalacia (PVL). PVL was defined as the presence of periventricular cysts on cranial ultrasound or cranial MRI scans. ROP was defined as $\geq$ stage 3 according to the International Classification of ROP (21). BPD was defined as mechanical ventilation or oxygen dependency at 36 weeks' postmenstrual age or discharge (22). Overall mortality was defined as the sum of in-hospital death and predicted death among DAMA infants, divided by the total number of infants.

\section{Statistical analysis}

Maternal and neonatal characteristics were compared using Student's $t$-tests for continuous variables and Chi-square tests for categorical variables. Number of participants with missing data for each variable of interest was indicated. A multivariable logistic regression model was used to calculate adjusted odds ratios (aOR). The covariates controlled for in this model included sex, gestational age, SGA, maternal hypertension, maternal diabetes. Antenatal steroids, Apgar score and TRIPS score were not included because they were intermediate variables that could affect the prognosis of inborn or outborn infants and may weaken the relationship between inborn or outborn status and prognosis. The covariates of our model were similar to recent publications (2). We repeated the analysis including steroids in the covariates of the multivariate model, the association between outborn status and mortality and brain injury remained significant. Sensitivity analysis was done among singleton infants, infants with birth weight $<1,500 \mathrm{~g}$ and infants who received complete care (excluding DAMA infants). We also used multilevel mixed-effects logistic regression models to examine the association of outborn status and neonatal outcomes accounting for the intracluster correlation among the infants admitted to same hospitals. Hospitals were considered as independent clusters with random effects in the models. A two-sided $\mathrm{P}$ value of $<0.05$ was used to determine statistical significance. Statistical analysis was performed using Stata 13.1 (StataCorp, 2013, College Station, TX, USA). 


\section{Results}

\section{Infant and maternal characteristics}

A total of 12,014 preterm infants born before 32 weeks' gestation were admitted to 18 perinatal centers in China during the study period from May 1st, 2015 to April 30th, 2018. Of these, $10,023(83.4 \%)$ infants were inborn, and $1,991(16.6 \%)$ infants were outborn. For infants born $<28$ weeks' gestation and $\geq 28$ weeks, the outborn rates were $15.6 \%(232 / 1,485)$ and $16.7 \%(1,759 / 10,529)$, respectively. Overall, $81.8 \%(1,629 / 1,991)$ infants were admitted to referral NICUs within 24 hours of life.

Infants and maternal characteristics are shown in Table 1. Outborn infants were less likely to receive prenatal care or be delivered by cesarean section, and had lower mean gestational age and Apgar scores but higher TRIPS score on admission compared with inborn infants (Table 1). Antenatal steroid use was significantly lower among outborn (46.3\%) infants compared with inborns (73.5\%) (Table 1).

\section{Outcomes}

Table 2 shows that the incidence of DAMA was significantly higher among outborn infants $(18.5 \%)$ compared with inborns $(12.5 \%, \mathrm{P}<0.001)$. In addition, the incidence of overall mortality $(19.9 \%$ vs. $15.8 \%, \mathrm{P}<0.001)$ and severe brain injury $(10.8 \%$ vs. $9.1 \%, \mathrm{P}=0.024)$ were higher in outborn infants than those of inborn infants. There were no differences in incidences of sepsis, NEC and severe ROP.

Table 3 shows that after adjustment, outborn status was significantly associated with a higher risk of DAMA (aOR, 1.6; $95 \%$ CI: $1.4-1.8$ ), overall mortality (aOR, $1.3 ; 95 \%$ CI: $1.1-1.5)$ and severe IVH or PVL (aOR, 1.2; 95\% CI: $1.0-1.5)$.

Sene Sensitivity analysis among singleton infants (Table S2), infants with birth weight $<1,500 \mathrm{~g}$ (Table S3) and infants who received complete care (Table S4) showed similar results. The multilevel mixed-effects logistic regression models accounting for the intracluster correlation among the infants admitted to same hospitals also showed similar results (Table S5).

\section{Discussion}

To our knowledge, this is the first report of outborn very preterm infant outcomes in China. Although our 16.6\% incidence of outborn infants among very preterm infants is similar to those reported by the Australian and New
Zealand Neonatal Network (13\%) and Canadian Neonatal Network (19\%) (10), it is likely to be an underestimate because we only considered outborns admitted to perinatal centers in China. We did not have data on delivery room deaths births in level I or level II hospitals who were not transferred, or admissions to NICUs at free standing children's hospitals. The latter are important because traditionally tertiary level NICUs are located at children's hospitals instead of perinatal centers in China, and they account for a significant proportion of VPIs. Despite the underestimation, our incidence of outborn infants $<28$ weeks' gestation (15.6\%) was significantly higher than some other developed countries like Japan (6.5\%) (23). Since infants with the lowest gestational age are at highest risk of adverse outcomes in outborn, our findings indicate an urgent need for the development of a regionalization program of perinatal care in China to reduce the incidence of outborn infants.

We found that the outborn infants had lower Apgar scores, and higher TRIPS score than inborn infants. This may be the result of differences in medical resources and expertise between tertiary perinatal centers and lower level perinatal centers. In addition, the stress of inter-hospital transfer on the infant may result in higher illness severity on NICU admission and be ultimately associated with adverse outcomes $(3,24,25)$. The incidence of maternal prenatal glucocorticoid use was significantly lower among outborn infants compared with inborns. One explanation may be the need for emergency delivery with resultant inadequate time for steroid administration. However, it is also possible that lack of staff awareness and routine practice guidelines for preterm births in lower level hospitals may also play important roles. Mothers of outborn infants were also less likely to have prenatal care and caesarean section, which may contribute to the higher incidence of adverse outcomes among these most vulnerable infants.

The risks of overall mortality and severe brain injury were also increased significantly among outborn infants compared with inborn infants. These results are consistent with previous studies $(3,5,8,9,23)$. In addition, outborn infants were associated with higher risk-adjusted probability of severe brain injury and overall mortality than inborn infants and may reflect sub-optimal resuscitation at the time of birth, lack of appropriate expertise to care for these infants after birth and sub-optimal transport. There was no significant difference in the incidence of BPD, sepsis, and ROP between outborn and inborn infants. Outborn infants were at lower risk of NEC but this may be because the most 
Table 1 Infant and maternal characteristics

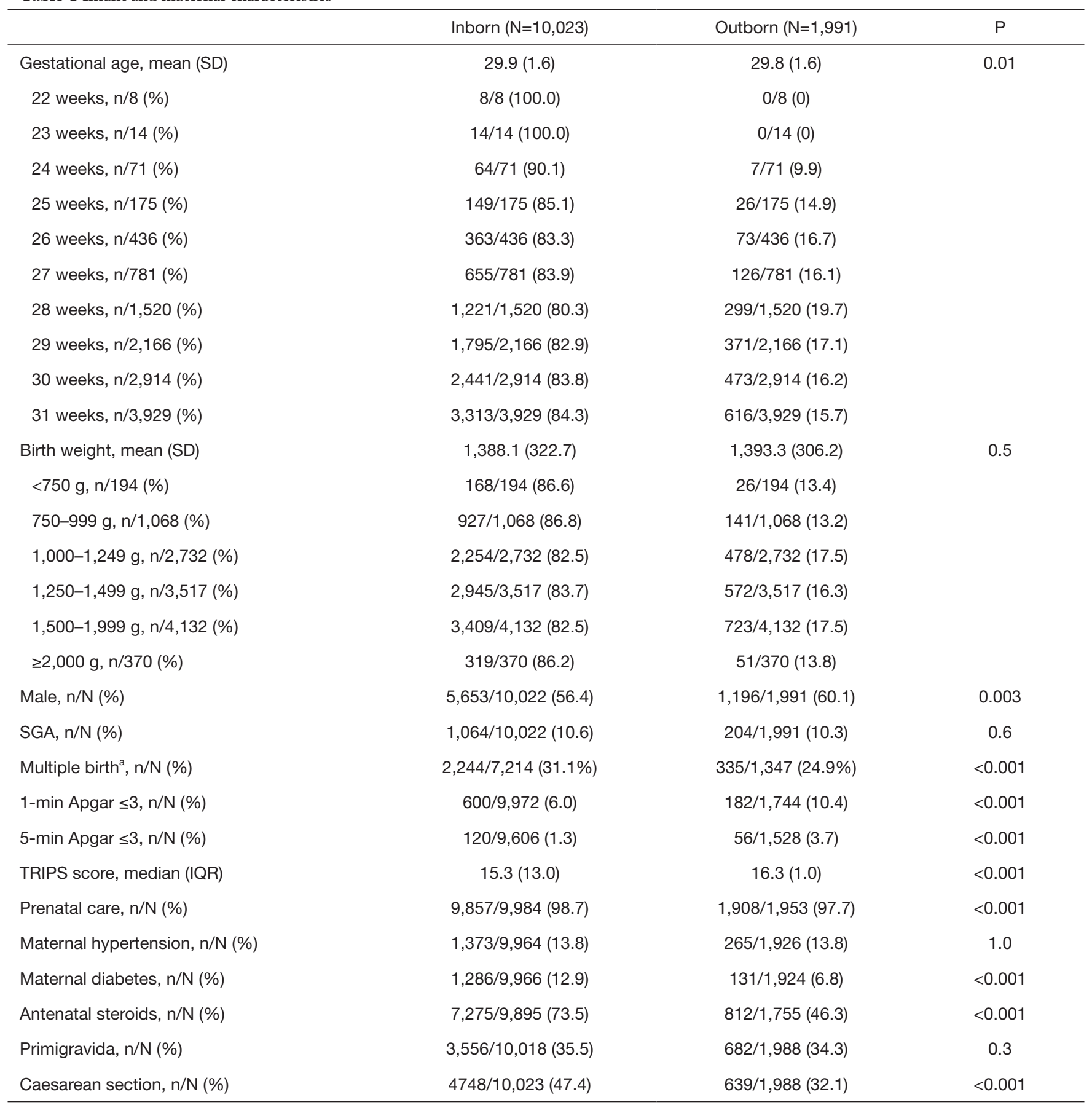

${ }^{a}$ Data on multiple birth was only collected during the last two years of study. SD, standard deviation; SGA, small for gestational age infant; TRIPS, Transport Risk Index of Physiologic Stability; IQR, interquartile range.

severely-ill outborn infants died at the early stage after birth and did not survive to develop these morbidities.

An important finding is that outborn infants were more likely to be DAMA and the majority of neonatal deaths among outborn infants occurred after DAMA. There might be several explanations for this. First, parents who chose to deliver at lower level hospitals may be less compliant with medical advice and more likely to have their infants 
Table 2 Comparison of outcomes for inborn and outborn preterm infants admitted to NICUs

\begin{tabular}{|c|c|c|c|}
\hline & Inborn $(\mathrm{N}=10,023)$ & Outborn $(\mathrm{N}=1,991)$ & $\mathrm{P}$ \\
\hline In-hospital mortality, n/N (\%) & $662 / 8,772(7.6)$ & $121 / 1,633(7.4)$ & 0.8 \\
\hline Overall mortality, $\mathrm{n} / \mathrm{N}(\%)^{\mathrm{b}}$ & $1,588 / 10,023(15.8)$ & $396 / 1,991$ (19.9) & $<0.001$ \\
\hline Sepsis, $\mathrm{n} / \mathrm{N}(\%)^{\mathrm{c}}$ & $741 / 10,023(7.4)$ & $153 / 1,991(7.7)$ & 0.6 \\
\hline IVH or PVL, n/N (\%) & $806 / 8,832(9.1)$ & $192 / 1,771(10.8)$ & 0.024 \\
\hline NEC, n/N (\%) ${ }^{\dagger}$ & $467 / 9,120(5.1)$ & $73 / 1,795$ (4.1) & 0.06 \\
\hline Severe ROP, n/N (\%) & $116 / 6,215(1.9)$ & $20 / 1,377(1.5)$ & 0.3 \\
\hline
\end{tabular}

${ }^{\mathrm{a}}$ In-hospital mortality = number of in-hospital death/number of infants who received active care. ${ }^{\mathrm{b}}$ Overall mortality $=$ (number of in-hospital

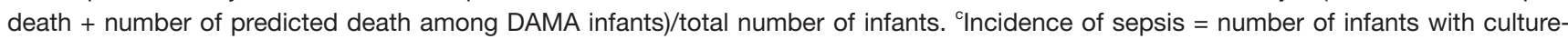
proven sepsis or clinical sepsis/number of all admissions. ${ }^{d}$ bronchopulmonary dysplasia. Incidence of BPD = number of infants who received active care and required mechanical ventilation or oxygen dependency at 36 weeks' postmenstrual age or discharge/number

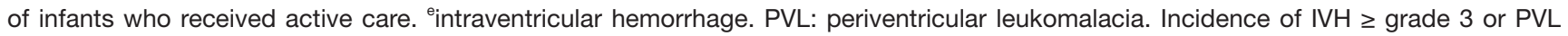
$=$ number of infants with $\mathrm{IVH} \geq$ grade 3 or PVL/number of infants with neuroimaging results. 'necrotizing enterocolitis. Incidence of NEC $=$ number of infants with NEC $\geq$ stage $2 /$ number of infants survived more than 72 hours. ${ }^{9}$ retinopathy of prematurity. Incidence of ROP = number of infants with ROP $\geq$ stage $3 /$ number of infants with eye examinations in NICU. DAMA, Discharge against medical advice; BPD, bronchopulmonary dysplasia; IVH, intraventricular hemorrhage; PVL, periventricular leukomalacia; NEC, necrotizing enterocolitis; ROP, retinopathy of prematurity.

Table 3 Crude and adjusted risks of morality and morbidities for outborn infants compared with inborn infants

\begin{tabular}{|c|c|c|c|c|c|c|}
\hline & \multicolumn{3}{|c|}{ Crude odds ratio } & \multicolumn{3}{|c|}{ Adjusted odds ratio ${ }^{a}$} \\
\hline DAMA & Reference & $1.5(1.3-1.7)$ & $<0.001$ & Reference & $1.6(1.4-1.8)$ & $<0.001$ \\
\hline In-hospital mortality & Reference & $1.0(0.8-1.2)$ & 0.847 & Reference & $1.0(0.8-1.2)$ & 0.842 \\
\hline Overall mortality & Reference & $1.3(1.2-1.5)$ & $<0.001$ & Reference & $1.3(1.1-1.5)$ & $<0.001$ \\
\hline BPD & Reference & $0.9(0.8-1.1)$ & 0.319 & Reference & $0.9(0.8-1.0)$ & 0.181 \\
\hline IVH or PVL & Reference & $1.2(1.0-1.4)$ & 0.024 & Reference & $1.2(1.0-1.5)$ & 0.012 \\
\hline NEC & Reference & $0.8(0.6-1.0)$ & 0.060 & Reference & $0.8(0.6-1.0)$ & 0.040 \\
\hline Severe ROP & Reference & $0.8(0.5-1.3)$ & 0.296 & Reference & $0.9(0.5-1.4)$ & 0.583 \\
\hline
\end{tabular}

${ }^{a}$ The covariates controlled for in this model included sex, gestational age, small for gestational age infant, maternal hypertension, maternal diabetes. DAMA, Discharge against medical advice; BPD, bronchopulmonary dysplasia; IVH, intraventricular hemorrhage; PVL, periventricular leukomalacia; NEC, necrotizing enterocolitis; ROP, retinopathy of prematurity.

DAMA. Second, parents may be concerned about the longterm prognosis of their infants. Previous authors reported that infants who were DAMA account for $53-92 \%$ of VPI deaths in Chinese NICUs and concern for long term adverse outcomes was the most common reason given for the decision to DAMA (13,26-32). Finally, a lack of appropriate prenatal and postnatal counselling might also contribute to DAMA. We speculate that if more VPIs could be delivered in tertiary perinatal centers with optimized parental consulting, the rate of DAMA may decrease.

There are some limitations of our study should be noted. We did not collect the information about the treatment received by outborn infants had in the delivery hospital, the infants who died in the delivery room and attitude of VPI 
delivery in referral hospitals. Also, we did not collect the information on the level of neonatal care in each referral hospital or the distance of transport. In addition, we also did not collect information regarding the socioeconomic or education status of mothers. Outborn infants admitted to free-standing hospitals were also not included in our study because characteristics of admitted infants as well as care practices are different between perinatal centers and children's hospitals. Therefore, our outborn rate might have been underestimated. Some of the infants in DAMA groups actually would have morbidities after discharge or did not survive to develop these morbidities, the overall risk for morbidities would be lower.

The incidence of outborn VPIs was high in China, especially among infants with lower gestational ages. They were at significantly higher risk of neonatal mortality and severe brain injury compared with inborn infants. Policies and quality improvement efforts are needed to facilitate inutero transfer of high-risk pregnancies to tertiary centers, and ultimately to improve the outcomes of VPIs in China.

\section{Acknowledgments}

The REIN-EPIQ Study Group: Yun Cao, Siyuan Jiang, Children's Hospital of Fudan Univeristy; Yong Ji, Children's Hospital of ShanXi / Women's Health Center of Shanxi, Shanxi, China; Shuping Han, Women's Hospital of Nanjing Medical University, Jiangsu, China; Sannan Wang, Suzhou Municipal Hospital, Jiangsu, China; Zhankui Li, Northwest Women and Children's Hospital, Shaanxi, China; Shiwen Xia, Women and Children's Hospital of Hubei Province, Hubei, China; Changyi Yang, Fujian Provincial Maternity and Children's Hospital/Affiliated Hospital of Fujian Medical University, Fujian, China; Chuanzhong Yang, The Affiliated Shenzhen Maternity and Child Healthcare Hospital of Southern Medical University, Guangdong, China; Ling Chen, Tongji Hospital, Tongji Medical College, Huazhong University of Science and Technology, Hubei, China; Ruobing Shan, Qingdao Women and Children's Hospital, Shandong, China; Ling Liu, Guiyang Maternal and Child Health Care Hospital, Guizhou, China; Bin Yi, Gansu Provincial Maternity and Child-care Hospital, Gansu, China; Zhenlang Lin, The $2^{\text {nd }}$ Affiliated Hospital and Yuying Children's Hospital of Wenzhou Medical University, Zhejiang, China; Yang Wang, The First Affiliated Hospital of Anhui Medical University, Anhui, China; Jiangqin Liu, Shanghai First Maternity and Infant Hospital, Tongji University School of Medicine, Shanghai,
China; Ling He, Jiangxi Provincial Children's Hospital, Jiangxi, China; Mingxia Li, MD, First Affiliated Hospital of Xinjiang Medical University, Xinjiang, China; Xinnian Pan, The Maternal and Child Health Hospital of Guangxi Zhuang Autonomous Region, Guangxi, China; Yan Guo, Children's Hospital of Nanjing Medical University, Jiangsu, China; Cuiqing Liu, Children's Hospital of Hebei Province, Hebei, China; Qin Zhou, The Affiliated Wuxi Maternity and Child Health Hospital of Nanjing Medical University, Jiangsu, China; Xiaoying Li, Qilu Children's Hospital of Shandong University, Shandong, China; Hong Xiong, Children's Hospital Affiliated to Zhengzhou University, Henan, China; Yujie Qi, Beijing Children's Hospital of Capital Medical University, Beijing, China; Mingyan Hei, The Third Xiangya Hospital of Central South University, Hunan, China.

Funding: This study was funded by the China Medical Board (Grant Number 14-194) and the Canadian Institutes of Health Research (Grant Number CTP 87518).

\section{Footnote}

Reporting Checklist: The authors have completed the STROBE reporting checklist. Available at http://dx.doi. org/10.21037/tp-20-232

Data Sharing Statement: Available at http://dx.doi. org/10.21037/tp-20-232

Peer Review File: Available at http://dx.doi.org/10.21037/tp20-232

Conflicts of Interest: All authors have completed the ICMJE uniform disclosure form (available at http://dx.doi. org/10.21037/tp-20-232). Dr. Shoo K. Lee serves as an unpaid editorial board member of Translational Pediatrics from Feb 2013 to Jan 2021. The other authors have no conflicts of interest to declare.

Ethical Statement: The authors are accountable for all aspects of the work in ensuring that questions related to the accuracy or integrity of any part of the work are appropriately investigated and resolved. The study was conducted in accordance with the Declaration of Helsinki (as revised in 2013). The study was approved by the Ethics Committee of the Children's Hospital of Fudan University (NO.201528) and written informed consent from the patients was not required because this study did not directly 
intervene in the diagnosis and treatment of individual patients.

Open Access Statement: This is an Open Access article distributed in accordance with the Creative Commons Attribution-NonCommercial-NoDerivs 4.0 International License (CC BY-NC-ND 4.0), which permits the noncommercial replication and distribution of the article with the strict proviso that no changes or edits are made and the original work is properly cited (including links to both the formal publication through the relevant DOI and the license). See: https://creativecommons.org/licenses/by-nc-nd/4.0/.

\section{References}

1. Blencowe H, Cousens S, Chou D, et al. Born too soon: the global epidemiology of 15 million preterm births. Reprod Health 2013;10 Suppl 1:S2.

2. Jensen EA, Lorch SA. Effects of a Birth Hospital's Neonatal Intensive Care Unit Level and Annual Volume of Very Low-Birth-Weight Infant Deliveries on Morbidity and Mortality. JAMA Pediatr 2015;169:e151906.

3. Helenius K, Longford N, Lehtonen L, et al. Association of early postnatal transfer and birth outside a tertiary hospital with mortality and severe brain injury in extremely preterm infants: observational cohort study with propensity score matching. BMJ 2019;367:15678.

4. Shah KP, deRegnier RO, Grobman WA, et al. Neonatal Mortality After Interhospital Transfer of Pregnant Women for Imminent Very Preterm Birth in Illinois. JAMA Pediatr 2020;174:358-65.

5. Chien LY, Whyte R, Aziz K, et al. Improved Outcome of Preterm Infants When Delivered in Tertiary Care Centers. Obstet Gynecol 2001;98:247-52.

6. Empana JP, Subtil D, Truffert P. In-hospital mortality of newborn infants born before 33 weeks of gestation depends on the initial level of neonatal care: the EPIPAGE study. Acta Paediatr 2003;92:346-51.

7. Rautava L, Eskelinen J, Hakkinen U, et al. 5-Year Morbidity Among Very Preterm Infants in Relation to Level of Hospital Care. JAMA Pediatr 2013;167:40-6.

8. Warner B, Musial MJ, Chenier T, et al. The Effect of Birth Hospital Type on the Outcome of Very Low Birth Weight Infants. Pediatrics 2004;113:35-41.

9. Lee SK, McMillan DD, Ohlsson A, et al. The benefit of preterm birth at tertiary care centers is related to gestational age. Am J Obstet Gynecol 2003;188:617-22.

10. Hossain S, Shah PS, Ye XY, et al. Outborns or Inborns:
Where Are the Differences? A Comparison Study of Very Preterm Neonatal Intensive Care Unit Infants Cared for in Australia and New Zealand and in Canada. Neonatology 2016;109:76-84.

11. American Academy of Pediatrics and the American College of Obstetrics and Gynecology. Guidelines for Perinatal Care. 5th ed. Washington, DC: American Academy of Pediatrics and the American College of Obstetrics and Gynecology 2002.

12. Zou L, Wang X, Ruan Y, et al. Preterm birth and neonatal mortality in China in 2011. Int J Gynaecol Obstet 2014;127:243-7.

13. Jiang S, Yan W, Li S, et al. Mortality and Morbidity in Infants <34 Weeks' Gestation in 25 NICUs in China: A Prospective Cohort Study. Front Pediatr 2020;8:33.

14. Lee SK, McMillan DD, Ohlsson A, et al. Variations in practice and outcomes in the Canadian NICU network: 1996-1997. Pediatrics 2000;106:1070-9.

15. Ballard JL, Novak KK, Driver M. A simplified score for assessment of fetal maturation of newly born infants. J Pediatr 1979;95:769-74.

16. Zhu L, Zhang R, Zhang S, et al. Chinese neonatal birth weight curve for different gestational age. Zhonghua Er Ke Za Zhi 2015;53:97-103.

17. Lee SK, Aziz K, Dunn M, et al. Transport Risk Index of Physiologic Stability, Version II (TRIPS-II): A Simple and Practical Neonatal Illness Severity Score. Am J Perinatol 2013;30:395-400.

18. Lee SK, Zupancic JAF, Pendray M, et al. Transport risk index of physiologic stability: A practical system for assessing infant transport care. J Pediatr 2001;139:220-6.

19. Stoll BJ, Hansen N, Fanaroff AA, et al. Late-onset sepsis in very low birth weight neonates: The experience of the NICHD Neonatal Research Network. Pediatrics 2002;110:285-91.

20. Papile LA, Burstein J, Burstein R, et al. Incidence and evolution of subependymal and hemorrhage: A study of infants with birth weights less than 1,500 gm. J Pediatr 1978;92:529-34.

21. International Committee for the Classification of Retinopathy of Prematurity. The international classification of retinopathy of prematurity revisited. Arch Ophthalmol 2005;123:991-9.

22. Jobe AH, Bancalari E. Bronchopulmonary dysplasia. Am J Respir Crit Care Med 2001;163:1723-9.

23. Sasaki Y, Ishikawa K, Yokoi A, et al. Short- and LongTerm Outcomes of Extremely Preterm Infants in Japan According to Outborn/Inborn Birth Status. Pediatr Crit 
Care Med 2019;20:963-9.

24. Palmer KG, Kronsberg SS, Barton BA, et al. Effect of inborn versus outborn delivery on clinical outcomes in ventilated preterm neonates: secondary results from the NEOPAIN trial. J Perinatol 2005;25:270-5.

25. Towers CV, Bonebrake R, Padilla G, et al. The effect of transport on the rate of severe intraventricular hemorrhage in very low birth weight infants. Obstet Gynecol 2000;95:291-5.

26. Zhang L, Qiu Y, Yi B, et al. Mortality of neonatal respiratory failure from Chinese northwest NICU network. Journal of Maternal-Fetal \& Neonatal Medicine 2017;30:2105-11.

27. Wang H, Gao X, Liu C, et al. Morbidity and mortality of neonatal respiratory failure in China: surfactant treatment in very immature infants. Pediatrics 2012;129:e731-740.

28. Ma L, Liu CQ, Wang YQ, et al. Mortality of Neonatal Respiratory Failure Related to Socioeconomic Factors in

Cite this article as: Pan S, Jiang S, Lin S, Lee SK, Cao Y, Lin Z; on behalf of the Reduction of Infection in Neonatal Intensive Care Units using the Evidence-based Practice for Improving Quality (REIN-EPIQ) Study Group. Outcome of very preterm infants delivered outside tertiary perinatal centers in China: a multi-center cohort study. Transl Pediatr 2021;10(2):306-314. doi: $10.21037 /$ tp-20-232
Hebei Province of China. Neonatology 2011;100:14-22.

29. Lin HJ, Du LZ, Ma XL, et al. Mortality and Morbidity of Extremely Low Birth Weight Infants in the Mainland of China: A Multi-center Study. Chinese Medical Journal 2015;128:2743-50.

30. Wang CH, Du LZ, Ma XL, et al. Analysis of In-hospital Neonatal Death in the Tertiary Neonatal Intensive Care Unit in China: A Multicenter Retrospective Study. Chinese Medical Journal 2016;129:2652-8.

31. Collaborative Study Group for Extremely Preterm \& Extremely Low Birth Weight Infants Collaborators. Survival and mortality rate of extremely preterm and extremely low birth weight infants admitted to neonatology departments. Chin J Pediatr 2014;52:729-35.

32. Wu F, Liu G, Feng Z, et al. Short-term outcomes of extremely preterm infants at discharge: a multicenter study from Guangdong province during 2008-2017. BMC Pediatr 2019;19:405. 
Supplementary

Table S1 Baseline characteristics of participating hospitals

\begin{tabular}{|c|c|c|c|c|c|c|c|c|}
\hline Hospital & $\begin{array}{l}\text { Inborn/ } \\
\text { Outborn }\end{array}$ & $\begin{array}{l}\text { Delivery/ } \\
\text { Year }\end{array}$ & $\begin{array}{l}\text { Teaching } \\
\text { Hospital }\end{array}$ & $\begin{array}{l}\text { NICU } \\
\text { Beds }\end{array}$ & $\begin{array}{l}\text { Intermediate/ } \\
\text { Continuing Care } \\
\text { Beds }\end{array}$ & $\begin{array}{l}\text { Number of } \\
\text { Neonatologists }\end{array}$ & $\begin{array}{l}\text { Number of } \\
\text { Nurses }\end{array}$ & $\begin{array}{l}\text { Transport } \\
\text { Team }\end{array}$ \\
\hline $\begin{array}{l}\text { The Maternal and Child Health } \\
\text { Hospital of Guangxi Zhuang } \\
\text { Autonomous Region }\end{array}$ & I/O & 16,000 & $\mathrm{~N}$ & 80 & 80 & 31 & 136 & $Y$ \\
\hline $\begin{array}{l}\text { Northwest Women and Children's } \\
\text { Hospital }\end{array}$ & I/O & 24,000 & $\mathrm{Y}$ & 50 & 150 & 37 & 138 & $Y$ \\
\hline $\begin{array}{l}\text { Gansu Provincial Maternity and Child- } \\
\text { care Hospital }\end{array}$ & $1 / O$ & 21,000 & $\mathrm{~N}$ & 70 & 150 & 9 & 101 & $Y$ \\
\hline $\begin{array}{l}\text { The Affiliated Wuxi Maternity and } \\
\text { Child Health Care Hospital of Nanjing } \\
\text { Medical University }\end{array}$ & I/O & 18,000 & $\mathrm{Y}$ & 20 & 40 & 20 & 48 & $\mathrm{~N}$ \\
\hline $\begin{array}{l}\text { Tongji Hospital,Tongji Medical } \\
\text { College,Huazhong University of } \\
\text { Scinece and Technology }\end{array}$ & I/O & 6,000 & $Y$ & 30 & 25 & 8 & 55 & $Y$ \\
\hline $\begin{array}{l}\text { First Affiliated Hospital of Xinjiang } \\
\text { Medical University }\end{array}$ & I/O & 6,000 & $Y$ & 30 & 15 & 9 & 34 & $\mathrm{Y}$ \\
\hline $\begin{array}{l}\text { The } 2^{\text {nd }} \text { Affiliated Hospital and Yuying } \\
\text { Children's Hospital of Wenzhou } \\
\text { Medical University }\end{array}$ & I/O & 11,820 & $\mathrm{Y}$ & 65 & 65 & 34 & 108 & $Y$ \\
\hline $\begin{array}{l}\text { The Affiliated Shenzhen Maternity and } \\
\text { Child Healthcare Hospital of Southern } \\
\text { Medical University }\end{array}$ & I/O & 19,793 & $\mathrm{Y}$ & 60 & 50 & 13 & 54 & $Y$ \\
\hline $\begin{array}{l}\text { The First Affiliated Hospital of Anhui } \\
\text { Medical University }\end{array}$ & I/O & 6,000 & $\mathrm{Y}$ & 20 & 40 & 11 & 43 & $\mathrm{~N}$ \\
\hline $\begin{array}{l}\text { Guiyang Maternal and Child Health } \\
\text { Care Hospital }\end{array}$ & I/O & 15,000 & $\mathrm{~N}$ & 70 & 65 & 40 & 93 & $\mathrm{~N}$ \\
\hline $\begin{array}{l}\text { The Third Xiangya Hospital of Central } \\
\text { South University }\end{array}$ & I/O & 4,000 & $\mathrm{Y}$ & 15 & 25 & 6 & 36 & $\mathrm{~N}$ \\
\hline Suzhou Municipal Hospital & $1 / 0$ & 18,000 & $\mathrm{Y}$ & 41 & 70 & 22 & 76 & $\mathrm{Y}$ \\
\hline
\end{tabular}

NICU, neonatal intensive care unit. 
Table S2 Crude and adjusted risks of morality and morbidities for outborn infants compared with inborn infants among singletons

\begin{tabular}{|c|c|c|c|c|c|c|}
\hline & \multicolumn{3}{|c|}{ Crude odds ratio } & \multicolumn{3}{|c|}{ Adjusted odds ratio ${ }^{a}$} \\
\hline DAMA & Reference & $1.7(1.4-2.0)$ & $<0.001$ & Reference & $1.7(1.4-2.0)$ & $<0.001$ \\
\hline In-hospital mortality & Reference & $0.9(0.7-1.2)$ & 0.543 & Reference & $0.9(0.7-1.2)$ & 0.511 \\
\hline Overall mortality & Reference & $1.4(1.1-1.6)$ & $<0.001$ & Reference & $1.4(1.1-1.6)$ & 0.001 \\
\hline BPD & Reference & $1.0(0.8-1.2)$ & 0.771 & Reference & $0.9(0.8-1.2)$ & 0.577 \\
\hline IVH or PVL & Reference & $1.3(1.1-1.7)$ & 0.015 & Reference & $1.3(1.1-1.7)$ & 0.015 \\
\hline NEC & Reference & $0.9(0.7-1.3)$ & 0.570 & Reference & $0.9(0.6-1.2)$ & 0.362 \\
\hline Severe ROP & Reference & $0.8(0.4-1.6)$ & 0.456 & Reference & $0.8(0.4-1.6)$ & 0.514 \\
\hline
\end{tabular}

${ }^{a}$ The covariates controlled for in this model included sex, gestational age, small for gestational age infant, maternal hypertension, maternal diabetes. DAMA, Discharge against medical advice; BPD, bronchopulmonary dysplasia; IVH, intraventricular hemorrhage; PVL, periventricular leukomalacia; NEC, necrotizing enterocolitis; ROP, retinopathy of prematurity.

Table S3 Crude and adjusted risks of morality and morbidities for outborn infants compared with inborn infants among infants $<1,500 \mathrm{~g}$

\begin{tabular}{|c|c|c|c|c|c|c|}
\hline & \multicolumn{3}{|c|}{ Crude odds ratio } & \multicolumn{3}{|c|}{ Adjusted odds ratio ${ }^{a}$} \\
\hline DAMA & Reference & $1.5(1.3-1.7)$ & $<0.001$ & Reference & $1.5(1.3-1.8)$ & $<0.001$ \\
\hline In-hospital mortality & Reference & $1.0(0.8-1.3)$ & 0.748 & Reference & $1.0(0.8-1.3)$ & 0.708 \\
\hline Overall mortality & Reference & $1.3(1.1-1.5)$ & $<0.001$ & Reference & $1.3(1.1-1.5)$ & $<0.001$ \\
\hline BPD & Reference & $0.9(0.8-1.1)$ & 0.528 & Reference & $0.9(0.8-1.1)$ & 0.278 \\
\hline IVH or PVL & Reference & $1.1(0.9-1.4)$ & 0.325 & Reference & $1.1(0.9-1.4)$ & 0.231 \\
\hline NEC & Reference & $0.8(0.6-1.1)$ & 0.136 & Reference & $0.8(0.6-1.1)$ & 0.121 \\
\hline Severe ROP & Reference & $0.7(0.4-1.2)$ & 0.243 & Reference & $0.8(0.4-1.3)$ & 0.345 \\
\hline
\end{tabular}

${ }^{a}$ The covariates controlled for in this model included sex, gestational age, small for gestational age infant, maternal hypertension, maternal diabetes. DAMA, Discharge against medical advice; BPD, bronchopulmonary dysplasia; IVH, intraventricular hemorraghe; PVL, periventricular leukomalacia; NEC, necrotizing enterocolitis; ROP, retinopathy of prematurity. 
Table S4 Crude and adjusted risks of morality and morbidities for outborn infants compared with inborn infants among infants received complete care

\begin{tabular}{|c|c|c|c|c|c|c|}
\hline & \multicolumn{3}{|c|}{ Crude odds ratio } & \multicolumn{3}{|c|}{ Adjusted odds ratio ${ }^{a}$} \\
\hline In-hospital mortality & Reference & $0.9(0.7-1.1)$ & 0.384 & Reference & $0.9(0.7-1.1)$ & 0.331 \\
\hline Sepsis & Reference & $1.1(0.9-1.3)$ & 0.613 & Reference & $1.0(0.9-1.3)$ & 0.692 \\
\hline BPD & Reference & $0.9(0.8-1.1)$ & 0.319 & Reference & $0.9(0.8-1.1)$ & 0.181 \\
\hline NEC & Reference & $0.7(0.4-1.6)$ & 0.159 & Reference & $0.8(0.6-1.0)$ & 0.072 \\
\hline Severe ROP & Reference & $1.0(0.8-1.2)$ & 0.842 & Reference & $0.8(0.5-1.3)$ & 0.390 \\
\hline
\end{tabular}

${ }^{a}$ The covariates controlled for in this model included sex, gestational age, small for gestational age infant, maternal hypertension, maternal diabetes. DAMA, Discharge against medical advice; BPD, bronchopulmonary dysplasia; IVH, intraventricular hemorrhage; PVL, periventricular leukomalacia; NEC, necrotizing enterocolitis; ROP, retinopathy of prematurity.

Table S5 Adjusted risks of morality and morbidities for outborn infants compared with inborn infants using multi-level logistic regression model

\begin{tabular}{llcl}
\hline & & Adjusted odds ratio & \\
\cline { 2 - 3 } & Inborn & Outborn & $<0.001$ \\
DAMA & Reference & $1.4(1.2-1.7)$ & 0.842 \\
In-hospital mortality & Reference & $1.0(0.8-1.2)$ & 0.019 \\
Overall mortality & Reference & $1.2(1.0-1.4)$ & 0.397 \\
Sepsis & Reference & $1.1(0.9-1.3)$ & 0.776 \\
BPD & Reference & $1.0(0.8-1.2)$ & 0.026 \\
IVH or PVL & Reference & $1.1(1.0-1.4)$ & 0.291 \\
NEC & Reference & $0.9(0.7-1.1)$ & 0.940 \\
Severe ROP & Reference & $1.0(0.6-1.7)$ & \\
\hline
\end{tabular}

Multilevel mixed-effects logistic regression models were used to examine the association of outborn status and neonatal outcomes accounting for the intracluster correlation among the infants within hospitals. Hospitals were considered as independent clusters with random effects in the models. At the infant level, we controlled for sex, gestational age, small for gestational age infant, maternal hypertension, maternal diabetes. DAMA, Discharge against medical advice; BPD, bronchopulmonary dysplasia; IVH, intraventricular hemorrhage; PVL, periventricular leukomalacia; NEC, necrotizing enterocolitis; ROP, retinopathy of prematurity. 